Revista de Matemática: Teoría y Aplicaciones 5(1): 113-124

UCR - CCSS ISSN: 1409-2433

\title{
SHAPLEY'S VALUE FOR FUZZY GAMES
}

\author{
Raúl Alvarado Sibaja*
}

Received: November 4, 1997

\begin{abstract}
This is the continuation of a previous article titled "Fuzzy Games", where I defined a new type of games based on the Multilinear extensions $f$, of characteristic functions $v$ for cooperative games. We showed that $f$ fulfills the definition of characteristic functions and most of standard theorems for cooperative games also hold for this new type of games: The fuzzy games. Now we give some other properties and the extension of the definition of Shapley's Value for Fuzzy Games
\end{abstract}

Keywords: game theory, fuzzy sets, multiattribute decisions.

\section{Resumen}

Esta es la continuación de un trabajo anterior titulado "Fuzzy Games", en donde se definió un nuevo tipo de juegos, basados en las extensiones multilineales $f$, de las funciones características $v$, de los juegos cooperativos. Mostramos ahí que $f$ satisface las condiciones de la definición de las funciones características, y que muchos de los teoremas básicos de la teoría de los juegos cooperativos también son válidos para este nuevo tipo de juegos: Los juegos borrosos. Ahora damos algunas otras propiedades y una extensión de la definición del valor de Shapley para los juegos borrosos.

Palabras clave: teoría de juegos, conjuntos difusos, decisiones multiatributos.

AMS Subject Classification: 90D06, 90D12, 90D35

\section{Introduction}

Sections 1 through 7 are just the smallest possible subset of the previous article, necessary to understand the rest of this article. We keep the same indexation of sections, definitions and propositions, in order to make easier the search of specific proofs in that article. Sections 8 and 9 are the answers to the tasks proposed for this article. Section 10 deals with the computational problems of the definition we give in section 9. Section 11 is a motivational example of fuzzy games.

${ }^{*}$ Escuela de Ciencias de la Computación e Informática, Universidad de Costa Rica, 2060 San José, Costa Rica. 


\section{Cooperative games}

Most definitions and properties can also be found in [Roberts, 1976] or [Owen,1982]

\section{Multilinear extensions}

Definition 2.1 (Owen,1982) The multilinear extension of $v, f$, is defined as:

$$
f\left(x_{1}, \ldots, x_{n}\right)=\sum_{S \subseteq I}\left\{\prod_{j \in S} x_{j} \cdot \prod_{j \notin S}\left(1-x_{j}\right)\right\} v(S), \text { for } 0 \leq x_{j} \leq 1, j=1, \ldots, n .
$$

\section{Formulation of the problem}

We showed that this function matches $v$ at the corners of the $n$-dimensional unitary cube (that is why it is an extension of $v$ ); but, what is the meaning of $f(x)$ when $x$ is an interior point of the cube? It seems that Owen was interested in this function $f$ for the purpose of proving Shapley's value formula by other means; he calls $x$ a random coalition. I think that a more appropriate name would be fuzzy coalition, since it is a "in between coalitions" object. If these points $x$ are the new type of coalition, and $f$ is an extension of $v$, an obvious question is if $f$ also extends the properties of $v$ to the interior of the cube, that is:

$$
f(x \cup y) \geq f(x)+f(y)) \text { if } x \cap y \neq \emptyset \text { ? (Property 1) } f(\emptyset)=0 \text { ? (Property 2) }
$$

\section{Fuzzy sets}

To prove these properties we need to state what we mean by fuzzy subset, union, intersection and empty set. We just follow the standard definitions of fuzzy set theory [Kaufmann, 1975].

Definition 4.1 Let $E$ be a set. Let $\mu$ be a function $\mu: E \rightarrow[0,1]$, a fuzzy subset $Z$ of $E$ is the set of pairs $Z=\{e, \mu(e)\}$ for all $e \in E$.

\section{Cooperative games extended to fuzzy coalitions}

Proposition 5.1 The second property holds. See [ALVARADO, 1996]

Proposition $5.2 f(x \cup y)=f(x+y)$. See [ALVARADO, 1996]

Proposition 5.3 $f(x+y) \geq f(x)+f(y)$. Proof in [OWEN, 1982] [ALVARADO,1987]

Proposition 5.4 From the preceding propositions follows the first property. [ALVARADO, 1996]

Definition 5.1 Let $\mu_{x}: E \longrightarrow[0,1]$, such that $\mu_{x}(j)=x_{j}$. Similarly $\mu_{y}(j)=y_{j}$. 
Now we can talk of basic properties of coalitions such as $x \subseteq y, x \cup y, x \cap y$. We prove that the function $\mathrm{f}$ fulfills the two basic properties given in section 3 .

Definition 5.2 Given a game $v$, and its multilinear extension $f$, we define the asociated fuzzy game as function $f$. Since it has the properties of characteristics functions, and the coalitions are the fuzzy sets, defined over the set of players $I$ of $v$.

\section{Other properties of fuzzy games}

Here we prove that most basic properties of cooperatives games also hold for fuzzy games.

Proposition $6.1 z$ is an imputation for game $v \Leftrightarrow z$ is an imputation for game $f$. [ALVARADO, 1996]

Proposition 6.2 $v$ is an essential game if and only if $f$ is an essential game. [ALVARADO,1996]

Proposition 6.3 If $v$ is constant sum then $f$ is constant sum. [ALVARADO, 1996]

Proposition 6.4 If $v$ is (0,1)-normalized then $f$ is (0,1)-normalized [ALVARADO, 1996].

Proposition 6.5 If $v$ is a 0 -normalized game then

a) $\mathrm{f}(x) \geq 0$ for all $x$ in the unitary cube

b) $x \geq y \Rightarrow f(x) \geq f(y)$. [ALVARADO, 1988].and [ALVARADO, 1996]

\section{An open problem}

The purpose in this section is to recapitulate what has been done up to this point, in order to decide what is the road ahead.

\section{Some other properties of fuzzy games}

In this section we show some other properties of fuzzy games; among them the ones we talked about in section 7 . Namely,

- fulfills definition properties (1.5) and (1.6)

- satisfies theorem 1.1

- satisfies theorem 1.2
- pending, a suitable definition needed

- pending, needs (1.5) and (1.6)

- pending, needs (1.5) and (1.6)

Proposition 8.1 Let $p$ be a permutation over $I=\{1, \ldots, n\}$ the set of all players. Then $f\left(x_{p(1)}, x_{p(2)}, \ldots, x_{p(n)}\right)=f\left(x_{1}, x_{2}, \ldots, x_{n}\right)$.

Proof. Obvious from the definition of Multiple Linear Extensions, since the permutation is just a relabeling of the set of players, as in standart cooperative games. 
Proposition $8.2 f\left(0, \ldots, 0, x_{i}, 0, \ldots, 0\right)=x_{i} v(i)$.

Proof. From the definition of MLE we can split the set of coalitions S into two classes. One is just the set $\mathrm{S}=\{\mathrm{i}\}$, the other class is the rest of the coalitions, hence

$$
f\left(0, \ldots, x_{i}, \ldots, 0\right)=\sum_{S \subseteq I, S \neq\{i\}}\left\{\prod_{j \in S} x_{j} \cdot \prod_{j \notin S}\left(1-x_{j}\right)\right\} v(S)+x_{i} v(i)
$$

Note that for every set $S$ in this sum there exist $j$ such that $j$ belongs to $S$ and then $x_{j}=0$, hence every term in the sum is null, that is

$$
f\left(0, \ldots, x_{i}, \ldots, 0\right)=0+x_{i} v(i) .
$$

Proposition 8.3 $f\left(x_{1}, x_{2}, \ldots, x_{n}\right) \geq \sum x_{i} v(i)$.

Proof. From Prop. (5.2) and iterative use of (5.3) we have

$$
f\left(x_{1}, x_{2}, \ldots, x_{n}\right)=f\left(\sum\left(0, \ldots, x_{i}, \ldots, 0\right)\right) \geq \sum f\left(0, \ldots, x_{i}, \ldots, 0\right)=\sum x_{i} v(i) .
$$

Definition 8.1 Let $x$ be a fuzzy coalition in $[0,1]^{n}$ the $n$-dimensional unit cube, let $0<\alpha \leq 1$. We define the set $x_{\alpha}=\left\{i \in I / x_{i} \geq \alpha\right\}$.

Definition 8.2 Let $w$ and $z$ be imputations, we say that $w$ is $\alpha$-preferred over $z$ by $x_{\alpha}$ if

$$
\begin{gathered}
\forall i \in x_{\alpha}: w_{i}>z_{i} \\
\sum w_{i} \leq f\left(x_{\alpha}\right) .
\end{gathered}
$$

Note that $f\left(x_{\alpha}\right)$. is just $v\left(x_{\alpha}\right)$, since $a_{\alpha}$. is a standard coalition. Also, when $\alpha=1$ this definition is exactly the one given in (1.5) and (1.6).

This must be so, because the set of imputations is the same for both games $v$ and $f$ (see Prop. 6.1). It should be this way since if one imputation dominates another for game $v$ the same must happens in game $f$ because $\mathrm{f}$ is an extension of $v$.

Definition 8.3 Let $w$ and $z$ be two imputations, we say that $w$ is $\alpha$-preferred over $z$ if there exist a coalition $x_{\alpha}$ such that $w$ is $\alpha$-preferred over $z$ by $x_{\alpha}$.

Proposition 8.4 The $\alpha$-core of the game consist of all nondominated imputations.

Before the next proposition, it should be point out that for any feasible value of $\alpha$, and any colalition $S$ in game $v$, and there are several fuzzy coalitions a such that $S=x_{\alpha}$ as can be easily seen, when $n=2$, in Fig. 8.1 . For example, given $\alpha$, for any point $x$ in the shaded area, $x_{\alpha}$ is just the coalition $S=\{1\}$

Proposition 8.5 The $\alpha$-core consist of all imputations $w$ such that

$$
\sum_{i \in x_{\alpha}} w_{i} \geq f\left(x_{\alpha}\right) \text { for all } x_{\alpha} \neq \emptyset
$$




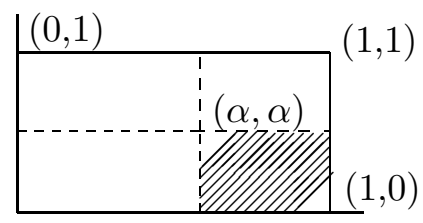

Figure 8.1

Proof: Obvious since any $x_{\alpha}=S$ for some $S \subseteq I$, and $f\left(x_{\alpha}\right)$. is just $v\left(x_{\alpha}\right)$.

Since we have Prop. 8.4 we can say that Theorem 1.1 also holds for fuzzy games.

Since the set of imputations is the same for both games $v$ and $f$, it is natural to ask if the the respectives concepts of domination are equivalent, that is:

Proposition 8.6 $w G z$ if and only if $w$ is $\alpha$-preferred over $z$.

Proof. In definition 8.2 we saw that if one imputation dominates another for game $v$ the same must happens in game $f$ because $\mathrm{f}$ is an extension of $v$. The other direction is also evident, since given a feasible value of $\alpha$ if (8.1) and (8.2) hold, the respective $S$ (see Fig. 8.1) satisfies (1.5) and (1.6).

Proposition 8.7 Theorem 1.2 also holds for fuzzy games.

Proof. Evident from Prop. 8.5

\section{The Shapley's value for fuzzy games}

In section 7 we saw that the problem of the Shapley's value for Fuzzy Games was still pending. Here we give an answer for that question. Before any attempt to define Shapley's value formula for function $\mathrm{f}$, we must be aware of the underlying differences.

Game $v$

- Set of players $I$ is finite, $|I|=n$

- Set of coalitions $2^{I}$ is finite, $\left|2^{I}\right|=2^{n}$

- $H_{i}(v)=\sum_{S \subseteq I} g(s)[v(S)-v(S-\{i\})]$
Game $f$

- Set of players $I$ is finite $|I|=n$

- Set of coalitions is the $n$-dimensional unit cube $C$, it is infinite and not numerable

The summatories must be transformed into integrals over suitable regions of the unit cube $C$. Also a suitable substitution for $g(s)$ must be found.

Looking to (1.16) more closely, we know that $v(S)$ must be somehow substituted by $f(x)$, but for every i we do not know the degree of belonging of $i$ to $S$, but what we know for sure is that $i$ it is not in $S-\{i\}$, hence we know that the substitute for $v(S-\{i\})$ must be such that it has 0 at the $i$-th component. Another problem is $g(s)$ since this is an integer valued function and $s$ is the number of members in coalition $S$, but how many members has coalition $x$ ?. A definition that meets the requirements for $\mathrm{g}$ is the following: 
Definition 9.1 Let $x$ be any for game $f$, let $s_{x}$ be

$$
s_{x}=\text { Integer of }\left[\sum_{i=1}^{n} x_{i}=v(I)+0.5\right] .
$$

Note that when $x$ is one of the corners of the unit cube $C$, that is, when $x$ coincides with some $S \subseteq I$, then $s_{x}=|S|=s$.

For example, for $n=4$, when $x$ is the corner $(0,1,1,1)$ then $s_{x}=\operatorname{Int}[0+1+1+1+0.5]=3$.

Definition 9.2 (Shapleys value for Fuzzy Games)

$$
H_{i}(f)=\int_{C} g\left(s_{x}\right)\left[f(x)-f\left(x_{1}, x_{2}, \ldots, x_{(i-1)}, 0, x_{(i+1)}, \ldots, x_{n}\right)\right] d x
$$

Notes: If we take only the corners of $C$ then $f(x)$ becomes $v(S)$ and $f\left(x_{1}, x_{2}, \ldots, x_{(i-1)}, 0, x_{(i+1)}, \ldots, x_{n}\right)$ becomes $v(S-\{i\})$. Also $g\left(s_{x}\right)=g(s)$, hence (9.2) is exactly (1.16).

Definition (9.2) is the answer to the question in section 7, and for theoretical purposes we can close our work here. But the task of solving that integral it is not an easy one. So

the objective of the next section is to give a more practical procedure to find the value of $(9.2)$

\section{Computational aspects of Shapley's value for fuzzy ga- mes}

We know that every cooperative game is equivalent (isomorphic) to a game in $(0,1)$ normalization. Hence, we will assume from now on that $v$ is in $(0,1)$ normalized.

Proposition $10.1 H_{i}(f) \leq 1$.

Proof: Obvious since $g\left(s_{x}\right) \leq 1$ and $f(x) \leq 1$ and $f\left(x_{1}, x_{2}, \ldots, x_{(i-1)}, 0, x_{(i+1)}, \ldots, x_{n}\right) \leq 1$.

Proposition $10.2 \int_{c} f(x) d x=\frac{1}{2^{n}} \sum_{S \subseteq I} v(S)$

ProOF:

$$
\begin{aligned}
\int_{c} f(x) d x & =\int_{c} \sum_{S \subseteq I} \prod_{j \in S} x_{j} \cdot \prod_{j \notin S}\left(1-x_{j}\right) v(S) d x \\
& =\sum_{S \subseteq I} v(S) \int_{c} \prod_{j \in S} x_{j} \cdot \prod_{j \notin S}\left(1-x_{j}\right) d x \\
& =\sum_{S \subseteq I} \prod_{j \in S}\left[\int_{0}^{1} x_{J} d x_{J}\right] \cdot \prod_{j \notin S} \int_{0}^{1}\left(1-x_{J}\right) d x_{J}
\end{aligned}
$$




$$
\begin{aligned}
& =\sum_{S \subseteq I} v(S) \cdot \prod_{j \in S}\left[\frac{x_{j}^{2}}{2}\right]_{0}^{1} \cdot \prod_{j \notin S}\left[x_{J}-\frac{x_{J}^{2}}{2}\right]_{0}^{1}=\sum_{S \subseteq I} v(S) \cdot \frac{1}{2^{s}} \cdot \frac{1}{2^{n-s}} \\
& =\sum_{S \subseteq I} v(S) \cdot \frac{1}{2^{n}}=\frac{1}{2^{n}} \sum_{S \subseteq I} v(S)
\end{aligned}
$$

\section{Proposition 10.3}

$$
\int_{C} f\left(x_{1}, x_{2}, \ldots, x_{(i-1)}, 0, x_{(i+1)}, \ldots, x_{n}\right) d x=\frac{1}{2^{n-1}} \sum_{S \subseteq I, i \notin S} v(S) .
$$

Proof: First note that, since $x_{i}=0$, for every $S \subset I$ such that $i \in S$, the corresponding term in Def. (2.1) is null. Hence

$$
\begin{aligned}
& \int_{c} f\left(x_{1}, x_{2}, \ldots, x_{(i-1)}, 0, x_{(i+1)}, \ldots, x_{n}\right) d x= \\
& =\int_{c} \sum_{S \subseteq I, i \notin S} \prod_{j \in S} x_{J} \cdot \prod_{j \notin S}\left(1-x_{J}\right) v(S) \\
& =\sum_{S \subset I, i \notin S} v(S) \prod_{j \in S}\left[\int_{0}^{1} x_{j} d x_{j}\right] \cdot \prod_{j \notin S}\left[\int_{0}^{1}\left(1-x_{J}\right) d x_{J}\right] \\
& =\sum_{S \subset I, i \notin S} v(S) \frac{1}{2^{s}} \cdot \frac{1}{2^{n-s-1}} \\
& =\frac{1}{2^{n-1}} \sum_{S \subset I, i \notin S} v(S)
\end{aligned}
$$

\section{Proposition 10.4}

$$
\int_{c}\left[f(x)-f\left(x_{1}, x_{2}, \ldots, x_{(i-1)}, 0, x_{(i+1)}, \ldots, x_{n}\right)\right] d x=\frac{1}{2^{n}} \sum_{S \subseteq I}[v(S)-v(S-\{i\})]
$$

Proof: Let us split the set of coalitions $\{S / S \subseteq I\}$ into two classes: $\{S / S \subseteq I\}=$ $\{S \subseteq I / i \notin S\} \cup\{S \cup\{i\}\}$. Then, by (10.3) and (10.2)

$$
\begin{aligned}
& \int_{c}\left[f(x)-f\left(x_{1}, x_{2}, \ldots, x_{(i-1)}, 0, x_{(i+1)}, \ldots, x_{n}\right)\right] d x= \\
& =\frac{v(S)}{2^{n}}-\sum_{S \subseteq I, i \notin S} \frac{v(S)}{2^{n-1}} \\
& =\sum_{S \cup\{i\}, i \notin S} \frac{v(S \cup\{i\})}{2^{n}}+\sum_{S \subseteq I, i \notin S} \frac{v(S)}{2^{n}}-\sum_{S \subseteq I, i \notin S} \frac{v(S)}{2^{n-1}} \\
& =\sum_{S \subseteq I, i \notin S} \frac{v(S \cup\{i\})+v(S)-2 v(S)}{2^{n}}
\end{aligned}
$$




$$
\begin{aligned}
& =\frac{1}{2^{n}} \sum_{S \subseteq I, i \notin S}[v(S \cup\{i\})-v(S)] \\
& =\frac{1}{2^{n}} \sum_{S \subseteq I}[v(S)-v(S-\{i\})]
\end{aligned}
$$

So far we "forgot" the $g\left(s_{x}\right)$ factor in definition (9.2). Doing that, we have seen that the calculation of the rest of the integral it is not so hard. In order to deal with the $g\left(s_{x}\right)$ factor we can take two approaches, both are based on the following fact. If we split the unit cube in suitable regions, then $g\left(s_{x}\right)$ is constant within each one of these regions. Also, since $g(k)=g(n-k+1)$ and there is too some simmetry about the "area" or "volume" of these $n$-dimensional regions; then as a first approach, it seems reasonable to substitute $g\left(s_{x}\right)$ by a constant $K_{i}$, and later to calculate the value of $K_{i}$. The second approach will be to try to calculate the exact value for definition (9.2), but as will be shown by an example this is harder as $n$ grows.

If we substitute $g\left(s_{x}\right)$ by $K_{i}$ in definition (9.2) then

$H_{i}(f) \cong K_{i} \int_{C}\left[f(x)-f\left(x_{1}, x_{2}, \ldots, x_{(i-1)}, 0, x_{(i+1)}, \ldots, x_{n}\right)\right] d x=\frac{K_{i}}{2^{n}} \sum_{S \subseteq I}[v(S)-v(S-\{i\})]$

What is the value of $K_{i}$ ? . Again, since $H_{i}(f)$ must be equal to $H_{i}(v)$ at the corners of $C$, then we sate the equation:

$$
\frac{K_{i}}{2^{n}} \sum_{S \subseteq I}[v(S)-v(S-\{i\})]=\sum_{S \subseteq I} g(s)[v(S)-v(S-\{i\})]
$$

From wich we can obtain the value of $K_{i}$.

The second approach is splitting the cube in several regions and to integrate (9.2) on each one of them. Just to show how hard it can be we will do it for the case $n=2$. From the next figure we can see that $g\left(s_{x}\right)$ is constant in 3 regions.

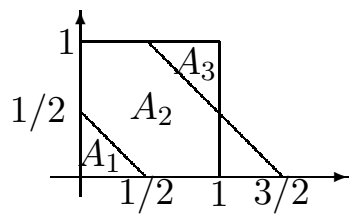

Figure 8.2

Note that $s_{x}=0$ for all $\mathrm{x}$ in region $A_{1}, s_{x}=1$ in region $A_{2}$ and $s_{x}=2$ in region $A_{3}$.

Note that for this example

$$
f(x)-f\left(0, x_{2}, \ldots, x_{n}\right)=f\left(x_{1}, x_{2}\right)-f\left(0, x_{2}\right)=x_{1}\left(1--x_{2}\right) v(1,0)+x_{1} x_{2} v(1,1) .
$$

Thus

$$
\begin{aligned}
H_{1}(f) & =\int_{C} g\left(s_{x}\right)\left[f(x)-f\left(x_{1}, x_{2}, \ldots, x_{(i-1)}, 0, x_{(i+1)}, \ldots, x_{n}\right)\right] d x \\
& =\int_{C} g\left(s_{x}\right)\left[f\left(x_{1}, x_{2}\right)-f\left(0, x_{2}\right)\right] d x_{1} d x_{2}
\end{aligned}
$$




$$
\begin{aligned}
= & \int_{0}^{\frac{1}{2}} \int_{0}^{\frac{1}{2}-x_{2}} g\left(s_{x}\right)\left[f\left(x_{1}, x_{2}\right)-f\left(0, x_{2}\right)\right] d x_{1} d x_{2} \\
& +\int_{\frac{1}{2}}^{1} \int_{\frac{1}{2}-x_{2}}^{1} g\left(s_{x}\right)\left[f\left(x_{1}, x_{2}\right)-f\left(0, x_{2}\right)\right] d x_{1} d x_{2}+ \\
& \int_{\frac{1}{2}}^{1} \int_{0}^{\frac{3}{2}-x_{2}} g\left(s_{x}\right)\left[f\left(x_{1}, x_{2}\right)-f\left(0, x_{2}\right)\right] d x_{1} d x_{2} \\
& +\int_{\frac{1}{2}}^{1} \int_{\frac{3}{2}-x_{2}}^{1} g\left(s_{x}\right)\left[f\left(x_{1}, x_{2}\right)-f\left(0, x_{2}\right)\right] d x_{1} d x_{2} \\
= & \int_{0}^{\frac{1}{2}} \int_{0}^{\frac{1}{2}-x_{2}} g(0)\left[f\left(x_{1}, x_{2}\right)-f\left(0, x_{2}\right)\right] d x_{1} d x_{2} \\
& +\int_{\frac{1}{2}}^{1} \int_{\frac{1}{2}-x_{2}}^{1} g(1)\left[f\left(x_{1}, x_{2}\right)-f\left(0, x_{2}\right)\right] d x_{1} d x_{2}+ \\
& \int_{\frac{1}{2}}^{1} \int_{0}^{\frac{3}{2}-x_{2}} g(1)\left[f\left(x_{1}, x_{2}\right)-f\left(0, x_{2}\right)\right] d x_{1} d x_{2} \\
& +\int_{\frac{1}{2}}^{1} \int_{\frac{3}{2}-x_{2}}^{1} g(2)\left[f\left(x_{1}, x_{2}\right)-f\left(0, x_{2}\right)\right] d x_{1} d x_{2} \\
= & g(0) \int_{0}^{\frac{1}{2}} \int_{0}^{\frac{1}{2}-x_{2}}\left[x_{1}\left(1-x_{2}\right) v(1,0)+x_{1} x_{2} v(1,1)\right] d x_{1} d x_{2}+ \\
& g(1) \int_{\frac{1}{2}}^{1} \int_{\frac{1}{2}-x_{2}}^{1}\left[x_{1}\left(1-x_{2}\right) v(1,0)+x_{1} x_{2} v(1,1)\right] d x_{1} d x_{2}+ \\
& g(1) \int_{\frac{1}{2}}^{1} \int_{0}^{\frac{3}{2}-x_{2}}\left[x_{1}\left(1-x_{2}\right) v(1,0)+x_{1} x_{2} v(1,1)\right] d x_{1} d x_{2}+ \\
& \text { tc. } \\
& \\
& \\
&
\end{aligned}
$$

For $n=3$ the cube will be split in 4 regions, but it will be necessary about 8 triple integral.

\section{An example of fuzzy games}

Game Theory is part of Conflict Resolution Theory, situations where some sort of bargaining is a must. In order to build an example of fuzzy games and of the Shapleys value for this type of games, we must first build an example of cooperative games. Unfortunately, interesting examples, even with small sets of players requires lots of calculations.

Let us say that five companies $A, B, C, D$ and $E$, are talking about a joint venture in a high risk, high technology business. Each company must invest $\$ 100$ million if the venture is carried out. This is a cooperative situation because they need the other players in order 
to make a profit; but it also a competitive situation, since all players surely will like to take the biggest share from the potential profits.

Before we continue with the example it is better define the notation we will use.

Usually a coalition or subset will be represented by a 5 -tuple, where 0 in the $\mathrm{i}^{\text {th }}$ position means the $\mathrm{i}^{\text {th }}$ player is it not in the coalition and 1 means it is part of the coalition. For example, the coalition $\{A, C\}$ will be represented as $(1,0,1,0,0)$. Sometimes will be easier to understand if we use a numerical index such as $1 \leq j \leq 5$.

The importance of the potential partners is not equal for all. Since companies $A$ and $B$ are the owners of vital know how, the joint venture cannot be done if one of them decides not to participate. This fact means several things:

$v(\{i\})=0$ for all $i, 1 \leq i \leq 5$.

$v(\{i, j\})=0$ for all $i, j, 1 \leq i, j \leq 5$, except maybe for $v(\{A, B\})$

$v(1,0,1,1,1)=v(0,1,1,1,1)=0$ and also is 0 for all subsets of these two coalitions.

Companies $A$ and $B$ cannot carry out the project just by themselves, so $v(\{A, B\})=0$.

The know how contribution of any of companies $C, D$ and $E$ (plus contribution of $A$ and $B$ of course), will be enough to carry out the project, but the final profit will vary according to who is in, the data is as follows:

$$
\begin{aligned}
& v(1,1,1,0,0)=0.65 \\
& v(1,1,0,1,0)=0.70 \\
& v(1,1,0,0,1)=0.75 \\
& v(1,1,1,1,0)=0.80 \\
& v(1,1,1,0,1)=0.85 \\
& v(1,1,0,1,1)=0.90 \\
& v(1,1,1,1,1)=1
\end{aligned}
$$

The function $v$ satisfies conditions 1.1 and 1.2 (the definition of cooperative games). As an specific example, we might think that the joint venture is to launch a telecommunications satellite and $A$ is the company who launches rockets, $B$ builds the satellites, $C$ is a video transmission company, $D$ a voice transmission company and $E$ a data transmission company.

Let us calculate Shapley's value for this game:

$$
\begin{aligned}
H_{A}(v)= & H_{B}(v)=g(3)[(0.65-0)+(0.70-0)+(0.75-0)]+g(4)[(0.80-0) \\
& +(0.85-0)+(0.90-0)]+g(5)[(1-0)]=g(3)[2.1]+g(4)[2.55]+g(5)[1] \\
= & 0.3975 \\
H_{C}(v)= & g(3)[(0.65-0)]+g(4)[(0.80-0.70)+(0.85-0.75)]+g(5)[(1-0.9)] \\
= & g(3)[0.65]+g(4)[0.2]+g(5)[0.1]=0.0683 \\
H_{D}(v)= & g(3)[(0.70-0)]+g(4)[(0.80-0.65)+(0.9-0.75)]+g(5)[(1-0.85)] \\
= & g(3)[0.7]+g(4)[0.3]+g(5)[0.15]=0.0716 \\
H_{E}(v)= & g(3)[(0.75-0)]+g(4)[(0.85-0.65)+(0.9-0.7)]+g(5)[(1-0.8)] \\
= & g(3)[0.7]+g(4)[0.4]+g(5)[0.2]=0.085 .
\end{aligned}
$$


So far so good, everything is standard cooperative game theory, each of the 32 possible coalitions is identified with one of the corners of the 5 dimensional unitary cube, and each company it is or it is not a member of a particular coalition.

But real life is often more complex; some partners might not really be fully committed to the project; they might be reluctant to share part of its knowledge with the other companies, they may be short of cash, they might be hesitant about the success of the project, etc, etc. How can we deal with these blurry conditions?

This it is not a probabilistic situation, this it is not a YES or NOT situation; there is no binomial distribution of probability, telling us that player $i$ belongs to a coalition with probability $x_{i}$ and not belonging with probability $\left(1-x_{i}\right)$

What we can do, is to build the following model: 0 means the company is out of the game, 1 means it is fully committed, any other number in $[0,1]$ express the degree of commitment to the project. But this is just the definition of fuzzy sets.

Notice that a 5 -tuple $\left(x_{1}, x_{2}, x_{3}, x_{4}, x_{5}\right)$ it is no longer a "random coalition". Now, all players are in every coalition, the difference is the level of belonging to any of them.

\section{Conclusions}

In section 10 we made two approaches to the computation of $H_{i}(f)$. The second approach will yield the exact value, but we showed that even for a small number of players, it is difficult to obtain a geneal (and easy) computational procedure. But the situation might not be as bad as it looks, for many real examples most of the coalitions $S$ are such that $v(S)=0$, and often there are many simmetries within subsets of players. All these factor are likely to cause great simplifications of expression (9.2)

\section{References}

[1] ALVARADO, R. (1980) "A game with a priori unions", Revista Ciencia y Tecnología 4(1,2): $21-36$.

[2] ALVARADO, R. (1985) "El problema del valor multiatributo.Una aproximación desde la teoría de juegos", Revista Ciencia y Tecnología 9(1,2): 7-10.

[3] ALVARADO, R. (1987) "Un algoritmo para la toma de decisiones en el caso de múltiples criterios", Revista Ciencia y Tecnología 11(1): 37-42.

[4] ALVARADO, R, (1992) "Multiattribute decision problems. An algorithm for long matrices", Revista Ingeniería 2(2): 11-25.

[5] ALVARADO, R. (1996) "Fuzzy games", Revista Ingeniería 1(5): 9-17.

[6] ALVARADO, R. (1991) Decision Support Systems Models Based on Multiattribute Utility Functions for Decision Making. Master of Science Thesis. University of South Carolina. 
[7] HWANG, C. AND YOON, K. (1981) Multiattribute Decision Methods and Applications. Lecture Notes in Economics and Mathematical Systems. Springer-Verlag, Berlin.

[8] FORSYTH, R. (1984) Expert Systems. Chapman and Hall, London

[9] KAUfmAnN, A. (1975) Theory of Fuzzy Sets. Academic Press, New York.

[10] OWEN, G. (1982) Game Theory. Second Edition. Academic Press, New York.

[11] ROBERTS, F. (1976) Discrete Mathematical Models. Prentice-Hall, New Jersey.

[12] TERANO, T. AND ASAI K. (1987) Fuzzy Systems Theory. Academic Press, Boston. 